\title{
Helix grounding electrode with good grounding performance
}

\author{
Zhanlong Zhang, Yihua Dan*, Daojun Mei, Jing Zou, and Peiyu Jiang \\ State Key Laboratory of Power Transmission Equipment \& System Security and New \\ Technology(Chongqing University), Chongqing, 400030, China
}

\begin{abstract}
Grounding electrode is used for discharging current and ensure the safe and stable operation of electrical equipment. Grounding performance of grounding electrode will degradation with limited installation space. This paper proposes helix grounding electrode and builds theoretically and simulating model on the helix grounding electrode. Analyzing grounding surface potential and step voltage distribution, grounding resistance and surface current density distribution. Results show helix grounding electrode can reduce grounding resistance, improve grounding surface potential distribution and the discharging current distribution.
\end{abstract}

\section{Introduction}

Grounding device buried in the soil is used to ensure the safe and stable operation of various electrical equipment. Grounding electrode is the core part of the grounding device, the current discharging performance of grounding electrode is an important indicator of grounding performance. Horizontal grounding electrode and vertical grounding electrode are the common grounding ways in the most project. Both grounding electrodes are affected by terrain easily, such as some location closed to the rock or river, the length and depth of the grounding electrode will be limited and causing the poor current discharging performance, grounding resistance increasing, and the operation can't meet the thermal stability requirements power accidents. Horizontal grounding electrode and the downlead are easy to be corroded in the soil. Especially the junction between the grounding electrode the downlead because of end-affect caused by non-uniform discharging current distribution. Corrosive breakage of the grounding electrode can cause serious power accidents.

Domestic and foreign scholars have made remarkable achievements in the research on the dispersion of grounding devices at present. Professor $\mathrm{Chen} \mathrm{Ci}$-xuan realized the calculation of the leakage current distribution by the grounding device through zero-order interpolation of the boundary element and the first-order interpolation of the boundary element, and the sub-section closed to the intersection is subdivided into much smaller two, more accurate current density distribution can be obtained [1]. To improve the analysis accuracy of the grounding device's current discharging characteristics, Wen Xishan established a mathematical model of vertically stratified soil structure for calculating the

\footnotetext{
*Corresponding author: microdanyihua@outlook.com
} 
grounding grids of large-scale water conservancy and hydropower plants [2]. These grounding grids are located in horizontal and vertical stratified soil with complex resistivity of soil. Water, underwater media and the soil resistivity across both sides of the straits are very different, a mathematical model that vertically divides the three-layer soil structure need to be established. The formula of ground current distribution is deduced by Laplace equation, which solves the problem of numerical calculation of vertical three-layer soil grounding parameters. Yuan Tao proposed "needle" grounding electrode from the concept of improving the impact current discharging efficiency of the grounding electrode and unevenness of the discharging impact current, effectively improve the resistance reduction characteristics of grounding electrode [3-5].

The above studies mainly focus on present grounding electrode, most scholars seldom studied the discharging current distribution characteristics of the other structure grounding electrode. Based on discharging current maximum concept, this paper proposed a helix grounding electrode. Theoretical calculation method for space current discharging characteristics, the surface current density, the end effect and the optimal buried depth range of the helix grounding electrode and ground surface potential distribution are analyzed.

\section{Theoretical analysis for helix discharging current}

Calculation and analysis of grounding electrode current discharging characteristics, widely used numerical methods such as the boundary element method, finite element method, but the above methods are too complicated. In order to simplify the computation, this paper mainly analyzes the low-frequency steady-state grounding characteristics of helix grounding electrode, uses electrostatic fields equivalent the current field in the soil and discharge the charge distribution on the surface of the grounding electrode to indicate current discharging performance of the grounding electrode. Analysis of the discharging current distribution of helix grounding electrode by moment method

Assuming that the helix grounding electrode is in an infinitely uniform soil and establishing the calculation model as shown in figure 1.

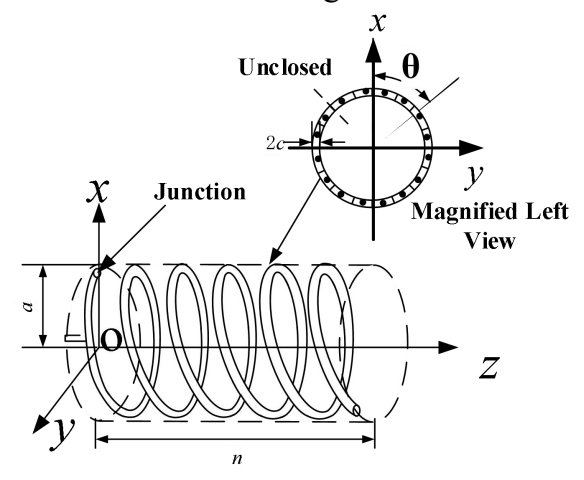

Fig. 1. Calculation model of helix grounding electrode.

As shown in figure 1, the major radius of helix grounding electrode is $a$, the minor radius of helix grounding electrode is $c$, the number of turns is $n$. The equal length of helix grounding electrode is $2 \pi a n \gg 2 c$, The distribution of surface charge density $\sigma\left(\boldsymbol{r}^{\prime}\right)$ of a helix grounding electrode can be regarded as the linear charge density $\tau\left(\boldsymbol{r}^{\prime}\right)$ along the spiral line of the grounding electrode. According to the analog charge method, the potential equation of the grounding electrode and the equivalent spiral line equation can be established as below. 


$$
\begin{gathered}
\int_{S} \tau(\boldsymbol{r})\left(4 \pi \varepsilon_{0}\left|\boldsymbol{r}-\boldsymbol{r}^{\prime}\right|\right)^{-1} d s=\varphi \\
\left\{\begin{array}{l}
x=a \cos \theta \\
y=a \sin \theta \\
z=b \theta
\end{array}\right.
\end{gathered}
$$

$S$ is the equal length of the helix grounding electrode, $\left|\boldsymbol{r}-\boldsymbol{r}^{\prime}\right|$ is the distance between the field point and the source point. Based on the idea of micro-element, the continuous line charge source $\boldsymbol{\tau}(\boldsymbol{r})$ within the one turn spiral grounding electrode is approximately divided into $m$ discrete point charge sources $\boldsymbol{\tau}_{\boldsymbol{i}}$ The point charge source $\boldsymbol{\tau}(\boldsymbol{r})$ is discretized to $\tau_{i}$ by the pulse function $N_{i}(\theta)$ on $\theta$.

$$
\boldsymbol{\tau}(\boldsymbol{r})=\sum_{i=1}^{N} N_{i}\left(\theta_{i}\right) \tau_{i}
$$

Establishing discrete point charge source integral equation by bringing equation (2) (3) into equation (1).

$$
\int_{\Delta \theta} N_{i}\left(\theta_{i}\right)\left|\boldsymbol{r}-\boldsymbol{r}^{\prime}\right|^{-1} d \theta \sum_{i=1}^{N} \tau_{i}=4 \pi \varepsilon_{0} \varphi\left(a^{2}+b^{2}\right)^{-1 / 2}
$$

According to the moment method, using Dirac function as the weight function and establishing a point matching equation.

$$
\sum_{i=1}^{N} \tau_{i} \int_{0}^{\theta_{\max }} \delta\left(\boldsymbol{r}-\boldsymbol{r}_{\boldsymbol{j}}^{\prime}\right) d \theta \int_{\Delta \theta} N_{i}\left(\theta_{i}\right)\left|\boldsymbol{r}-\boldsymbol{r}^{\prime}\right|^{-1} d \theta=4 \pi \varepsilon_{0} \varphi\left(a^{2}+b^{2}\right)^{-1 / 2} \int_{0}^{\theta_{\max }} \delta\left(\boldsymbol{r}-\boldsymbol{r}_{\boldsymbol{j}}^{\prime}\right) d \theta
$$

Equation (5) is changed into matrix form.

$$
\mathbf{K} \cdot \boldsymbol{\tau}=\mathbf{C}
$$

$\tau$ is a column of to-be-solved vectors consisting of $\mathrm{N}$ point charge source $\tau_{i}$ as we can see from equation (5). Grounding electrode is usually made of good conductor such as copper or galvanized steel to ensure the effect of current discharging. This paper focus on the current discharging characteristic of grounding electrode in power frequency and steady state condition, potential $\varphi$ of grounding electrode can be approximated as a constant.

Ground potential $\varphi$ can be measured by the fall of potential method, $\mathrm{C}$ is a known vector. Charge distribution of the grounding electrode can be given by solving the coefficient matrix K. According to the equation (5) and equation (6), the equation can be obtained.

$$
\mathbf{K}=\int_{\Delta \theta} N_{i}(\theta)\left|\boldsymbol{r}_{j}^{\prime}-\boldsymbol{r}_{i}^{\prime}\right|^{-1} d \theta
$$

1) $j \neq i$, matching point and the source point are not in the same segment

In order to select the coordinates of the matching point on the surface of the grounding electrode, and the coordinates of the points of the charged points of the discrete grounding electrode, the equation (2) is taken into the equation of the coefficient matrix. $\boldsymbol{r}_{j}^{\prime}$ is the selected matching point coordinates of the grounding electrode surface, $\boldsymbol{r}_{\boldsymbol{i}}^{\prime}$ is the selected source point coordinates of the grounding electrode surface, element $k_{j i}$ in the matrix $\mathrm{K}$ can be solved through equation (2) and equation (7).

$$
k_{j i}=\int_{\theta_{i}-\frac{\Delta \theta}{2}}^{\theta_{i}+\frac{\Delta \theta}{2}}\left\{b^{2}\left(\theta_{j}-\theta\right)^{2}+2 a^{2}\left[1-\cos \left(\theta_{j}-\theta\right)\right]\right\}^{-1 / 2} d \theta
$$

2) $j=i$, matching point and the source point are in the same segment 
A much smaller segment of a helix grounding electrode with a circular section is equivalent to a cylinder to ensure a higher calculation accuracy, and a model is established as shown in figure 2 .

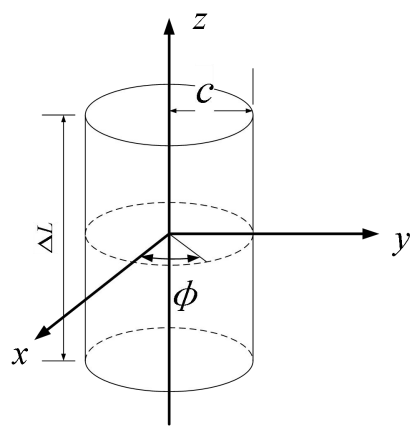

Fig. 2. Calculation model of coefficient.

As shown in figure 2, with the source of the segment $\boldsymbol{i}$ as the origin coordinates, the matching segment $\boldsymbol{j}$ is selected on the surface of the grounding electrode. Surface charge density $\sigma$ of the grounding electrode need to be solved first, then solving the surface discharging current. The micro segment area of the grounding electrode as below.

$$
\Delta \mathrm{A}=2 \pi \cdot c \cdot \Delta L
$$

Surface charge density $\sigma$.

$$
\sigma=\tau_{i}(\Delta L)\left(\Delta \mathrm{A}^{-1}\right)=\tau_{i}(2 \pi c)^{-1}
$$

Potential in centred point $I_{i}$ caused by surface current can be obtained through integrating the entire surface area A when the surface charge density of the grounding electrode is known.

$$
\begin{aligned}
\phi_{j} & =(4 \pi \varepsilon)^{-1} \int_{\Delta S} \sigma \cdot \vec{R}^{-1} d S=(4 \pi \varepsilon)^{-1} \cdot \int_{0}^{2 \pi} \int_{-\frac{\Delta L}{2}}^{\frac{\Delta L}{2}} c \cdot \tau_{i} \cdot(2 \pi c)^{-1} \cdot\left(c^{2}+z^{\prime 2}\right)^{-1 / 2} d z^{\prime} d \varphi \\
& =(4 \pi \varepsilon)^{-1} \cdot \tau_{i} \cdot 2 \cdot \ln \left\{c^{-1} \cdot\left[\Delta L / 2+\left(c^{2}+(\Delta L / 2)^{2}\right)^{1 / 2}\right]\right\}
\end{aligned}
$$

Whereas $\Delta L=\Delta \theta * a$, simplified equation (11) as below.

$$
\tau_{i} \cdot 2\left(a^{2}+b^{2}\right)^{-1 / 2} \cdot \ln \left\{c^{-1} \cdot\left[\Delta L / 2+\left(c^{2}+(\Delta L / 2)^{2}\right)^{1 / 2}\right]\right\}=4 \pi \varepsilon \phi_{j}\left(a^{2}+b^{2}\right)^{-1 / 2}
$$

Comparing equation (5) and equation (12).

$$
k_{j i}=2 c \cdot\left(a^{2}+b^{2}\right)^{-1 / 2} \cdot \ln \left\{c^{-1} \cdot\left[\Delta L / 2+\left(c^{2}+(\Delta L / 2)^{2}\right)^{1 / 2}\right]\right\}
$$

Solving the coefficient matrix $\mathbf{K}$ through equation (8) and equation (13), point charge $\tau_{i}$ distribution on helix grounding electrode surface can be solved. To solve the charge distribution of helix grounding electrode surface, each discrete segment $\tau_{i}$ is normalized $\tau_{i} /|\boldsymbol{\tau}|$, the charge distribution ratio at each point on the helix grounding electrode surface can be obtained. The current is the charge passing some section of the conductor in a unit time, so the discharging current distribution of the helix grounding electrode can be approximated by the distribution of charge distribution. 


\section{Analysis of the discharging current on helix grounding electrode}

Current discharging characteristic is one of the most important factors to measure grounding performance of grounding electrode. From the aspects of surface potential distribution, step voltage, grounding resistance, end effects, surface current density distribution, the length and buried depth of the helix grounding electrode, the current discharging characteristic of the helix grounding electrode is comprehensively analyzed.

\subsection{Analysis of the discharging current on helix grounding electrode}

Frequency of the grounding current is usually power frequency, and the grounding current field can be approximated as constant electric field, so the 1A DC is taken as power source in this simulation. Building a simulation model as shown in figure 3, Assuming that the turns of the helix grounding electrode is 20 , the minor radius is $0.02 \mathrm{~m}$, the axial pitch is $0.5 \mathrm{~m}$, the major radius $0.5 \mathrm{~m}$, and installing grounding electrodes along the $x$-axis. To compare the current discharging characteristic of helix grounding electrode and horizontal grounding electrode. Establishing horizontal grounding electrode model with the same axial installation length $(10 \mathrm{~m})$ and $0.02 \mathrm{~m}$ cross-section radius to comparative analysis, buried depth is $0.8 \mathrm{~m}$, soil resistivity is $200 \Omega \cdot \mathrm{M}$, radius of the equivalent model of the infinite soil is $50 \mathrm{~m}$.
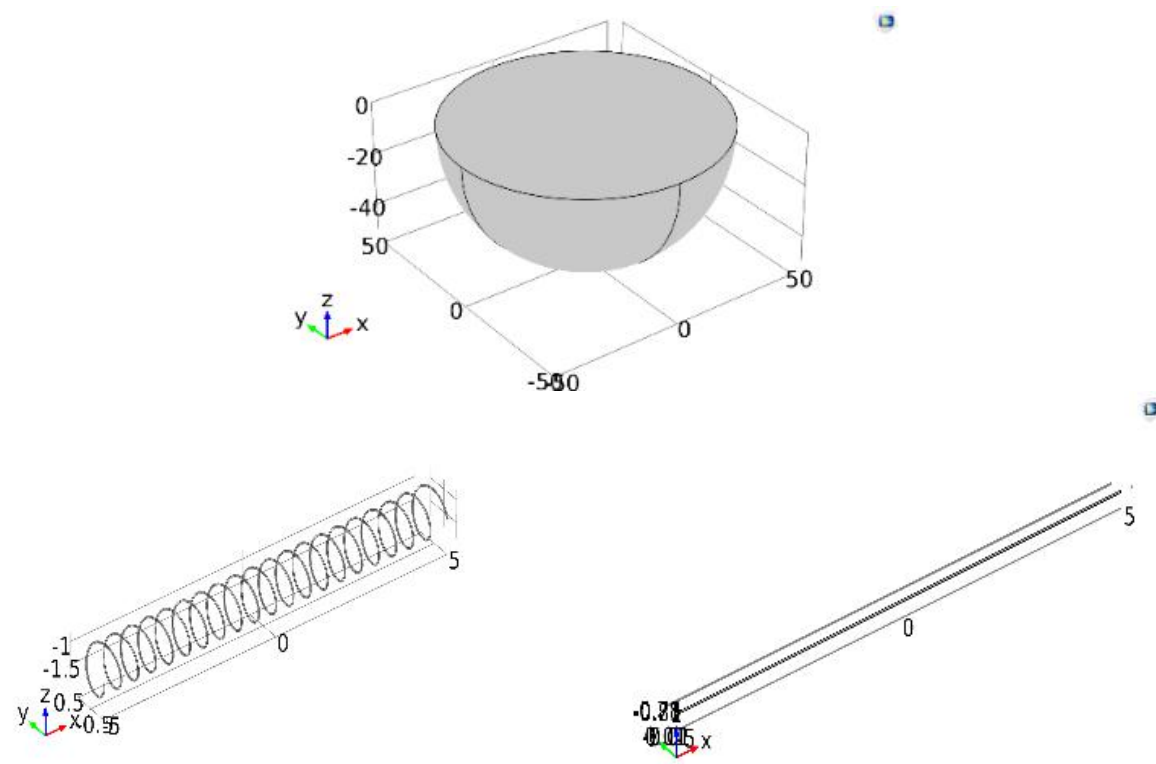

Fig. 3. Simulation Model Schematic.

\subsection{Ground surface potential distribution and step voltage}

Electric field will be generated in the soil surrounding the grounding electrode because the current is injected into the grounding electrode. The ground surface potential distribution above the grounding electrode is as shown below. 


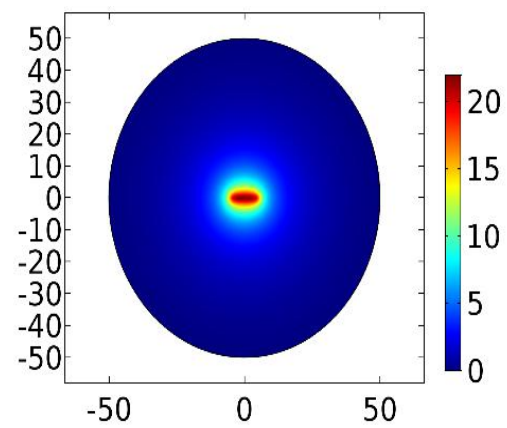

(a) Surface

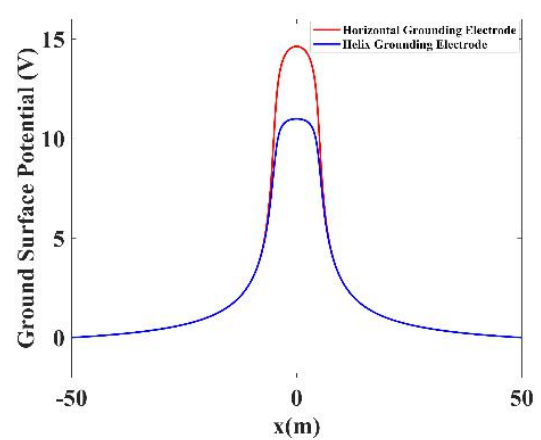

(b) $x$-axis direction

Fig. 4. Surface Potential Distribution.

Figure 4(a) shows that the surface potential above the helix grounding electrode appears an elliptical potential distribution pattern. Helix grounding electrode reduces the potential soil area effectively and electromagnetic interference caused by grounding electrode for surrounding environment. Figure 3(b) shows the maximum potential on the ground surface is $29.6 \mathrm{~V}$ in buried horizontal grounding electrode condition, the maximum potential on the ground surface is $21.9 \mathrm{~V}$ in buried helix grounding electrode condition. The maximum difference between the within the two inflection points of the curve is $26.01 \%$, the distribution are almost the same outside the two inflection points of the curve. Results indicates helix grounding electrode effectively reduces the step voltage around the inflection point of the curve.

The soil resistivity in actual projects is easily affected by the external environment. To analyse the current discharging performance of helix grounding electrode more comprehensively, the resistivity to (step length is $50 \Omega \cdot \mathrm{M}$ ) of soil are selected. Ground surface potential distribution is as follows in the different soil resistivity condition.
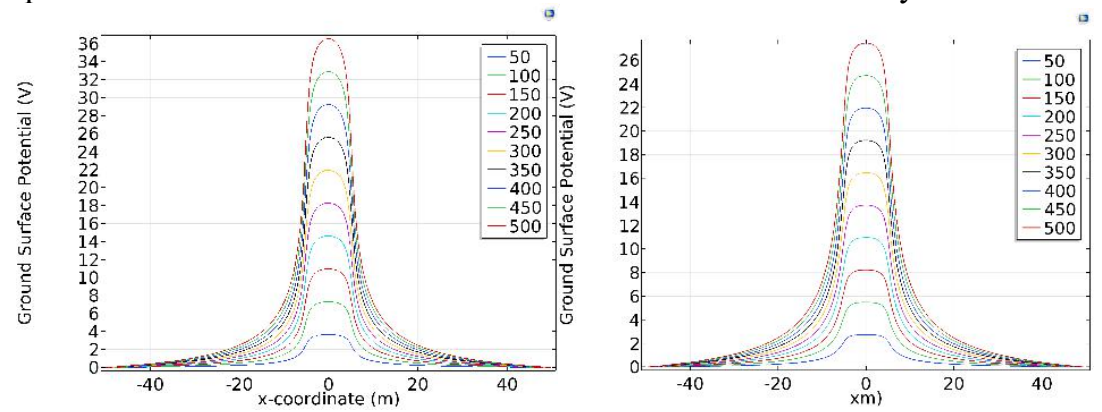

(a) Horizontal Grounding Electrode

(b) Helix Grounding Electrode

Fig. 5. Ground Surface Potential Distribution in Different Resistivity Soil.

Figure 5 shows that as the resistivity of the soil decreases, the maximum potential on the ground surface of helix grounding electrode and the horizontal grounding electrode both show a decreasing trend, and step voltage decreases at the inflection point of the curve ( both ends of the grounding electrode), ground surface area affected by the grounding electrode is also gradually reduced. The maximum ground potential rise of the helix grounding electrode is lower than about $25 \%$ of the horizontal grounding electrode at the same resistivity soil. The step voltage is significantly improved. Results indicate that the helix grounding electrode can achieve a better ground potential distribution in any resistivity soil. 


\subsection{Grounding Resistance}

Grounding resistance is an important indicator to measure the current discharging performance of helix grounding electrode. Power source is selected as 1A DC in this simulation, grounding electrode can be considered as a good conductor in the low frequency steady state field, the potential on grounding electrode surface is equal. So the potential value on grounding electrode surface means grounding resistance, the potential distribution on grounding electrode surface as shown below.

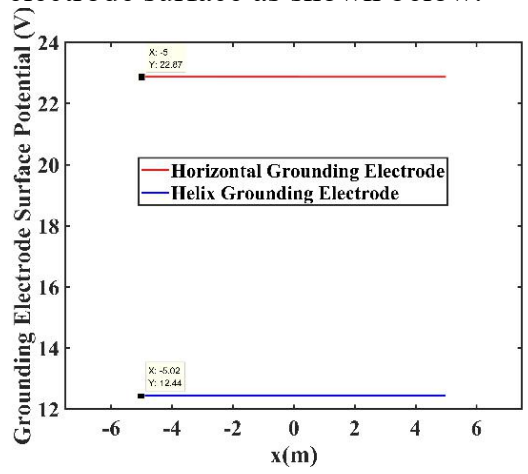

Fig. 6. Potential Distribution on Grounding Electrode Surface.

Figure 6 shows that the grounding resistance of the helix grounding electrode, and the resistance of the helix grounding electrode is in the same axial installation length. Grounding resistance of the helix grounding electrode was reduced by $45.6 \%$ compared to the same axial installation length of the horizontal grounding electrode. When the installation space is limited, helix grounding electrode maximizes the current discharging performance, greatly reducing the grounding resistance and improving the grounding performance.

To study the grounding resistance of grounding electrode in different resistivity soil, to soil resistivity (step length) is chose to analyze the ground performance, potential distribution on grounding electrode surface in different soil resistivity as shown below.

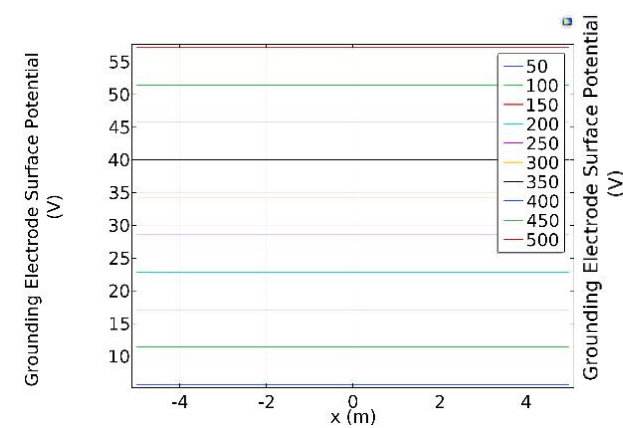

(a) Horizontal Grounding Electrode

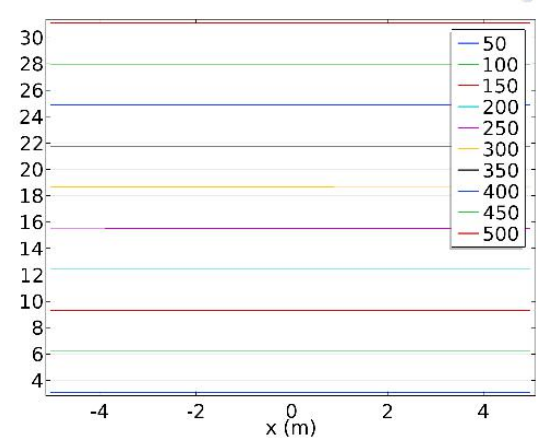

(b) Helix Grounding Electrode

Fig. 7. Potential Distribution on Grounding Electrode Surface in Different Soil Resistivity.

Figure 7 shows that grounding resistance of the grounding electrode tends to increase with the soil resistivity increasing. At the same resistivity, the grounding resistance of the helix grounding electrode is consistently lower than about $46 \%$ of the grounding resistance of the horizontal grounding electrode. On the one hand, helix grounding electrode has better current discharging characteristics and smaller grounding resistance in any resistivity soil, On the other hand, helix grounding electrode can ensure a relatively lower touch voltage and the safety of the operator when fault current flow into grounding electrode. 


\subsection{Discharging current distribution on helix grounding electrode surface}

Further study on the discharging current distribution on the helix grounding electrode surface, the discharging current distribution on the helix grounding electrode surface is as follow.

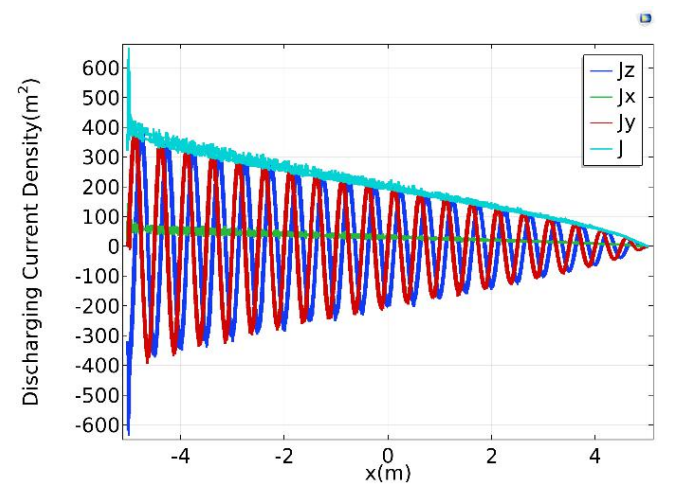

Fig. 8. Discharging Current Density Distribution on Helix Grounding electrode.

Figure 8 shows the $y$-axis component and the z-axis component of discharging current density on helix grounding electrode exhibit a decaying sinusoidal function distribution. With the structure of the helix grounding electrode, it can be seen that the effective current discharging length of the grounding electrode is increased under the same axial installation space. The linear attenuation of the discharging current density on the helix grounding electrode surface indicate that the helix grounding electrode has a good current discharging performance.

\section{Conclusion}

This paper proposes a helix grounding electrode based on the concept of maximizes the grounding current discharging. The following conclusions are drawn through extensive analysis.

1) Compared with the horizontal grounding electrode with the same axial installation length, the helix grounding electrode can effectively improve the surface potential distribution, reduce the step voltage and reduce the grounding resistance by about $45.6 \%$ in any resistivity soil.

2) Helix grounding electrode increases the contact area with the soil effectively and ensure a better discharging current distribution on the helix grounding electrode surface. The grounding performance of helix grounding electrode has obvious advantage over the horizontal grounding electrode.

In summary, the helix grounding electrode has good current discharging characteristics and grounding performance in low-frequency steady-state electrical field. So helix grounding electrode has far-reaching engineering significance with installation space consideration.

\section{Acknowledgments}

This work was supported by the National Natural Science Foundation of China (51577017).

\section{References}


1. Chen Ci-xuan. Application of Boundary Element Method in Ground CalculationCalculation of lightning protection grounding resistance [J]. High Voltage Engineering, 1983(2):15-25.

2. Wen Xishan, Dai Chuanyou, Fangyu. Computation of Current Field in a Vertical Three-layer Earth Structure[J], Journal of Wuhan University (Engineering Edition), 1997(1):53-56.

3. Tao Y. Analysis of Grounding Resistance Reduction Effect Based on Enhancing Impulse Current Leakage Efficiency[J]. Transactions of China Electrotechnical Society, 2012, 27(11):278-284.

4. Zhu Bing. Structure Parameter optimization of Grounding Device With NeedleShaped Conductors Based on Electric Field Distribution in Soil[J], Power System Technology, 2015, 39(10):2907-2914.

5. Li Jingli. Simulation and Experiment Study on Resistance-Reducing Mechanism ofGrounding Device With Spicules[J]. Power System Technology, 2013, 37(1):211217. 